\title{
Discover the Wonderful World of Plants with the Help of Smart Devices
}

\author{
Cosmin Irimia, Mihai Costandache, Mădălin Matei, Matei Lipan, \\ Ştefan Romanescu, Adrian Iftene \\ Faculty of Computer Science, “Alexandru Ioan-Cuza” University of Iaşi, Romania \\ \{cosmin.irimia, mihai.costandache, andrei.matei, radu.lipan, \\ stefan.romanescu, adiftene\} @info.uaic.ro
}

DOI: 10.37789/rochi.2020.1.1.12

\begin{abstract}
"Augmented Reality in Botanical Garden" (ARGB) is the name of the application we developed, which brings visitors closer to the Botanical Garden "Anastasie Fătu" from Iași. Even if they want to search for a plant to know where they can find it or maybe they are interested in knowing the garden and its sections, visitors can just access the app and get all the help they need. More than this, the app provides enhanced features for the people responsible for the maintenance of the garden such as the possibility to generate placards for different plants during different plant exhibitions.
\end{abstract}

\section{Author Keywords}

Android; iOS; Mobile app; Web app; Maps; Orientation and movement outside buildings.

\section{ACM Classification Keywords}

H.5.2. Information interfaces and presentation (e.g., HCI): User Interfaces. H.3.2. Information Storage and Retrieval: Information Storage.

\section{General Terms}

Human Factors; Design.

\section{INTRODUCTION}

Many people believe that computers improve our daily lives, but in many ways, computers, phones, and gadgets that surround us do nothing but isolate us further.

Probably the best example to support this is Virtual Reality (VR) where the big "benefit" is that the real world is replaced by an imaginary, virtual, computer-generated one where you can do almost what you want and be who you want. Instead, Augmented Reality (AR) uses computers to increase the richness of the real world [1], [4-6], [9-14]. It differs from virtual reality in that it does not attempt to replace the real one but only to add various improvements to it.
Our prototype guide through the Iasi Botanical Garden overlaps various information over the real world depending on the place and the images captured by the user's phone. This method will provide additional information than the tables already around the ecosystem built in the Botanical Garden. Besides information about each plant found in the garden, it also allows the identification of bird sounds and can even guide users through predetermined routes, designed to provide a better experience.

\section{EXISTING SOLUTIONS}

\section{PlantSnap - Plant Identifier}

PlantSnap ${ }^{1}$ is an iOS application that allows only through a simple photo to identify up to 625,000 plants and trees and provides information about them in over 30 languages (see Figure 1).

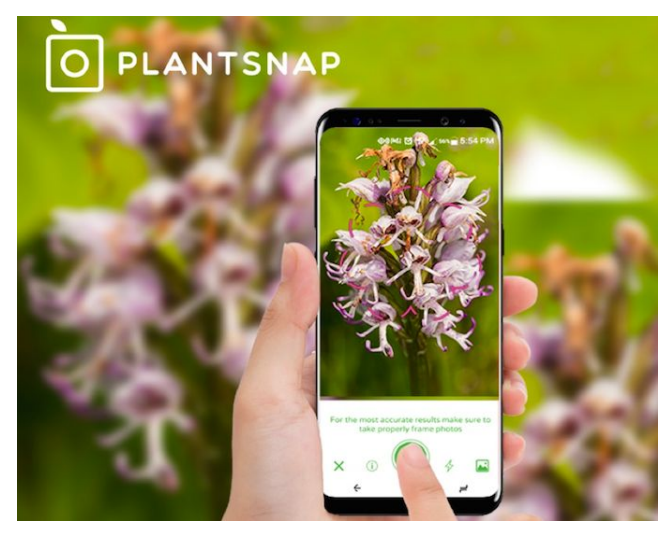

Figure 1. PlantSnap Application

Compared to our application, it has a much larger database

\footnotetext{
${ }^{1} \mathrm{https}$ //play.google.com/store/apps/details?id=com.fws.pla ntsnap2
} 
because the number of plants growing in the Iasi Botanical Garden is much smaller, but it does not offer any part of the AR for garden paths nor the identification of bird sounds.

\section{Garden Compass Plant/Disease Identifier ${ }^{2}$}

This is one of the best plant identification apps, as it does double duty: it can identify both the plant itself and assist you in identifying diseases that may be plaguing it (see Figure 2). As noted in some of our other problem-related articles, it is very important to identify diseases properly in order to deal with them appropriately, without causing more harm to the plant.

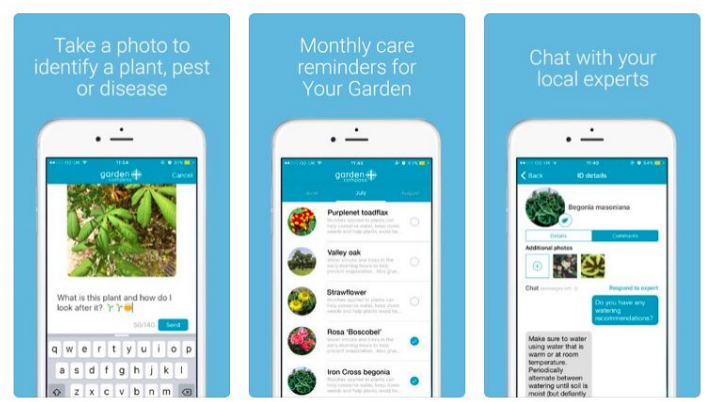

Figure 2. Garden Compass Plant Application

This app is useful, both for amateurs and experts, especially when it comes to combating diseases, and has an interesting technology built in but will not rise at the level of expectation we have with our needs.

\section{PictureThis - Plant Identifier}

PictureThis $^{3}$ is also an iOS application.

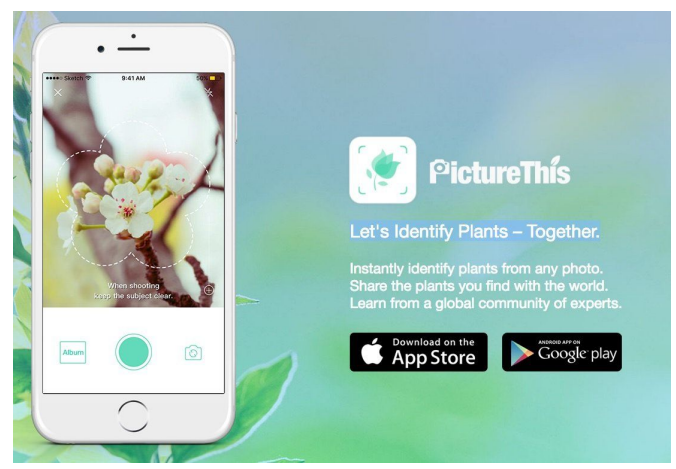

Figure 3. PictureThis Application

This allows with a simple picture to identify plants and trees, similar to the Plant Snap app, but has a much smaller

\footnotetext{
2 https://www.farms.com/agriculture-apps/gardening/garde n-compass-plant-disease-identifier

3 https://apps.apple.com/us/app/picturethis-plant-identifier/ id1252497129
}

database (see Figure 3). This can provide information, tell the user how to care for that plant and more than that, it allows posting pictures with plants in the community so anyone can use them as a wallpaper.

Apart from the fact that it does not offer a sound identification part, this application is prepaid, a big disadvantage compared to the one we built.

\section{BirdNET: Bird sound identification}

BirdNET $^{4}$ is an Android application that allows the user to continuously record the sounds of the environment, and select a piece of sound to predict which species of bird to sing (see Figure 4).
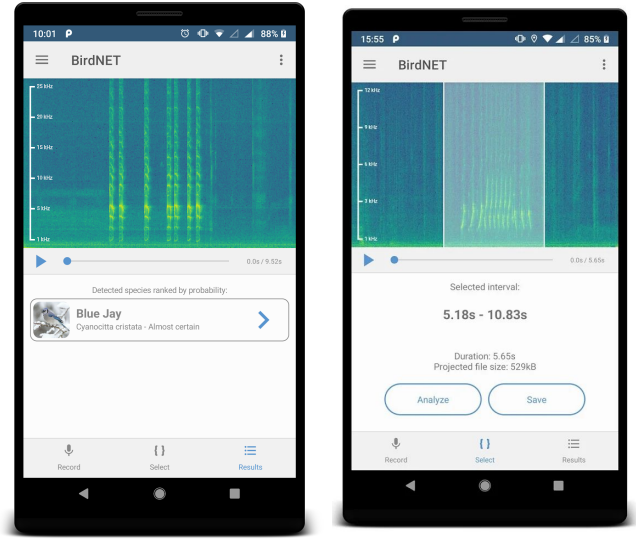

Figure 4. BirdNET Application

The application is free but it lacks many things. For example, it can not automatically identify the sound of the birds in the recording, the user has to select only a small piece, and also records the sounds in the environment continuously, which is a privacy issue.

\section{EXISTING WORK}

In [8], the authors analyzed the functions of botanical gardens, classified 30 different botanical gardens from all around the world according to purposes ordered by priority and studied the recreational functions of the Nezahat Gökyiğit Botanical Garden in Turkey. Several surveys on the topic of botanical gardens visitors' preferences are referenced by the authors and they also conducted their own. The authors were interested in aspects such as the seasons preferred by the visitors, the frequency of the visits or the reasons people enjoy being in a botanical garden.

In [3], the authors noticed that botanical gardens often focus on biological purposes, e.g., the need to preserve biodiversity, giving less importance to social and

\footnotetext{
${ }^{4}$ https://play.google.com/store/apps/details?id=de.tu_chem nitz.mi.kahst.birdnet\&hl=en_US
} 
educational aims. Educationally speaking, emotions and cognition together bring great benefits to the learning experience. There was proposed the concept of "useful botanical garden", based on three dimensions: sensibility (features that lead to welcoming comfort, safety and homeostasis), functionality (features that encourage interactions and cognitive processes) and rationality (visitors' reflection on values such as care and authority and how the values relate to the elements present in the botanical garden and the visitors' emotions). The presented ideas have implications for the design of botanical garden spaces and for the formative process based on garden spaces, visitor expectations and interactive activities.

In [7], the author approached the role of Mediterranean botanical gardens in the plant life of the region. They facilitate studies, introduction of new material, conservation and recovery and reintroduction of threatened species. The author noticed an imbalanced distribution of botanical gardens; more specifically, in the southern and eastern Mediterranean countries the botanical gardens have a less important role. Other problems were also analyzed, such as the fact that some botanical gardens cannot accommodate conservation collections of growing plants because they are too old and/or small. Several solutions to the identified problems were proposed.

In [2], the author described how the botanical gardens contribute to university education. The botanical gardens present diverse collections of plants and are accessible for frequent visits. Some skills in botany and/or related fields of activity are best taught with living plants, which make a botanical garden a great choice for learning (even if several activities that are performed in natural areas are not available here). Botanical gardens are also relaxing spaces that encourage learning and creativity. The author noted that the botanical gardens are underutilized, due to several reasons, e.g., proximity issues, lacking or inaccurate interpretation and labels.

\section{FUNCTIONAL REQUIREMENTS}

The functional requirements were established together with the experts from Botanical Garden of Iași, as they know best what problems they encounter in their work and also they can anticipate pretty accurately what a visitor wants. A main feature of the proposed solution from this paper is the capability of the system to store and collect different data for a series of plants. This part will be done with the help of an web app component:

- An employee from botanical garden will be delegated to insert data about the garden's plants;

- The employee will log in using an administrative account in the web application;
- In the application the user can add a new plant using the following properties: Plant's name, species, sub-species in latin, a description, qr code that will be near plant plate and an expiration time to make this plant inactive;

- After all the mandatory fields have been completed the user can save the record;

- The user can use the application to display a list with all of the records, to edit and delete them.
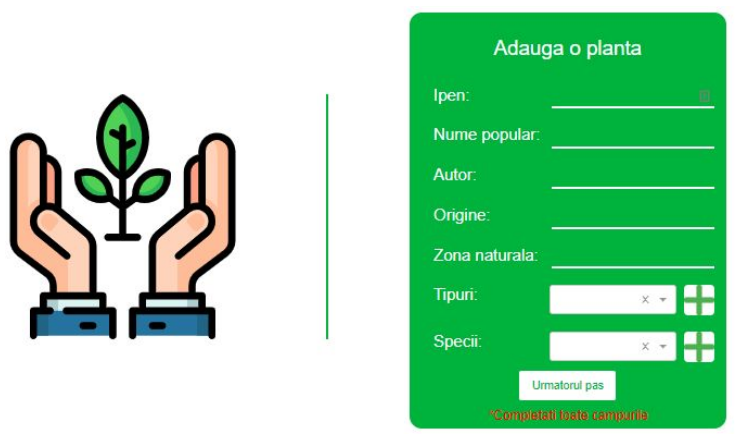

Figure 5. Web Application Component

Another component that will be delivered for visitors with the help of the garden's employee will be the one that guides them through the garden. This will be done with a mobile application component:

- An employee opens the app in admin mode;

- He/She opens "AR path generator" feature;

- The app opens the camera and detects the writing;

- With this map the app draws steps towards a tree or a plant;

- The admin saves the path and makes it accessible for the visitors.

The interaction between the garden's environment and the visitors will be made through a mobile application. The application is supported both on Android and iOS devices. A capability of this application will be to identify plants or trees with or without an QR code attached (see Figure 6). This will be done as follow:

- The user should install, firstly, the application from the dedicated marked (Google play or App Store);

- The user opens the application, selects from the menu "QR detector" and points the phone's camera to a QR code or to plant;

- After the image is processed, the user can see the description entered by the garden's employee or from the service that identifies images.

Apart from flowers, plants and trees the user can interact with the birds from the botanical garden. This is done as: 
- The user opens the application;

- Selects from the menu "Bird sound detection" feature and starts a sound recording session;

- Sends the recording to the Bird Recognition API;

- The API responds with the identified species (and perhaps other useful information);

- The user sends feedback regarding the received information, if he/she wants.

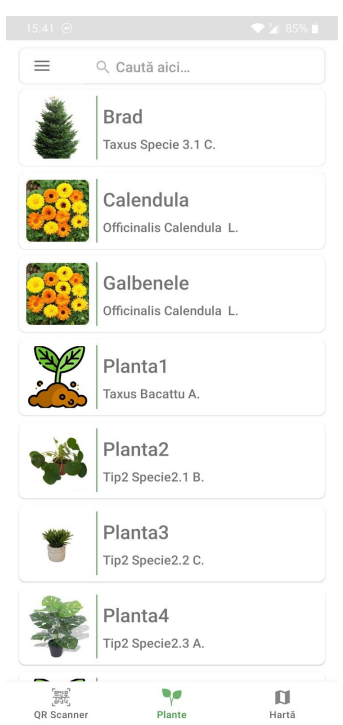

Figure 6. Mobile Application

The feedback feature is a possible feature, we will think about adding it. The main idea is that if the user is a specialist/bird enthusiast, he/she might know if the API is correct or not regarding a certain audio recording. The feedback could be used by the API. For example, an administrator checks the received feedback and if the user is correct, his/her response is used to improve the trained model. Another possible use would be to compute various metrics. Again, it would be recommended to have the users' responses checked by an admin.

\section{NON-FUNCTIONAL REQUIREMENTS}

Besides the functional requirements this application should fulfill non-functional requirements in order to be used efficiently. Thinking of these requirements usually implies technical knowledge, but the experts from the Botanical Garden of Iaşi also helped, as they are aware of the basic requirements of such a complex app too.

\section{Performance}

The application should respond fast enough to the image and sound recognition taking in consideration that the number of visitors in botanical gardens is growing every year. This should take into account the number of flower/trees species.

\section{Security}

Security measures should be taken into consideration in the admin mode when an employee enters data, so an external attacker will not alterate anything.

\section{Scalability}

Given the fact that a feature like bird sound recognition can be used much more frequently than others this should be easy to scale.

\section{Recoverability}

Because the path recording is a time consuming process for garden's employees there should be a way to recover these in case of a failure (e.g regular back ups).

\section{PROPOSED SOLUTION}

The ARGB Application has 4 main modules. The system architecture is presented in Figure 7.

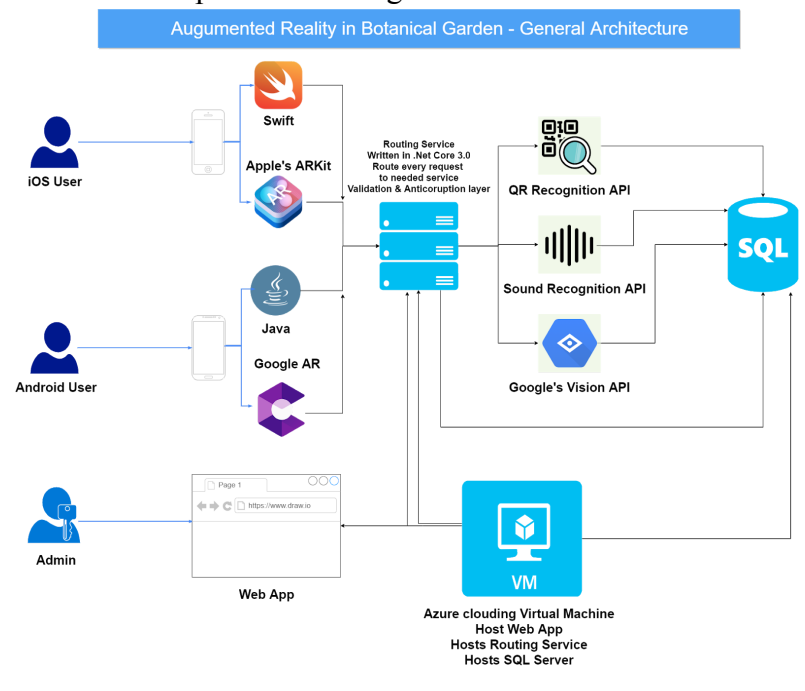

Figure 7. "ARGB" System Architecture

Each of these modules is presented in detail in the following paragraphs.

\section{Android App}

This module is the Android part of our application, it is written in Java and builded for phones that run Android $5^{5}$ (Lollipop) or higher (see Figure 8). When the app will be opened the user will get a prompt asking for Camera Permission. If users deny, the app will close itself, else the app will start and will search for an AR Core App installed on the phone. If the user does not have it already installed,

\footnotetext{
${ }^{5}$ https://www.android.com/versions/lollipop-5-0/
} 
it will get redirected to Google Play Store to install it. If the AR Core is already present, the app will start just fine. In the diagram below, we show the states of the app during the start operation. After the app opens, the user has a camera view right in front of his eyes and with this, he/she can explore the surroundings.

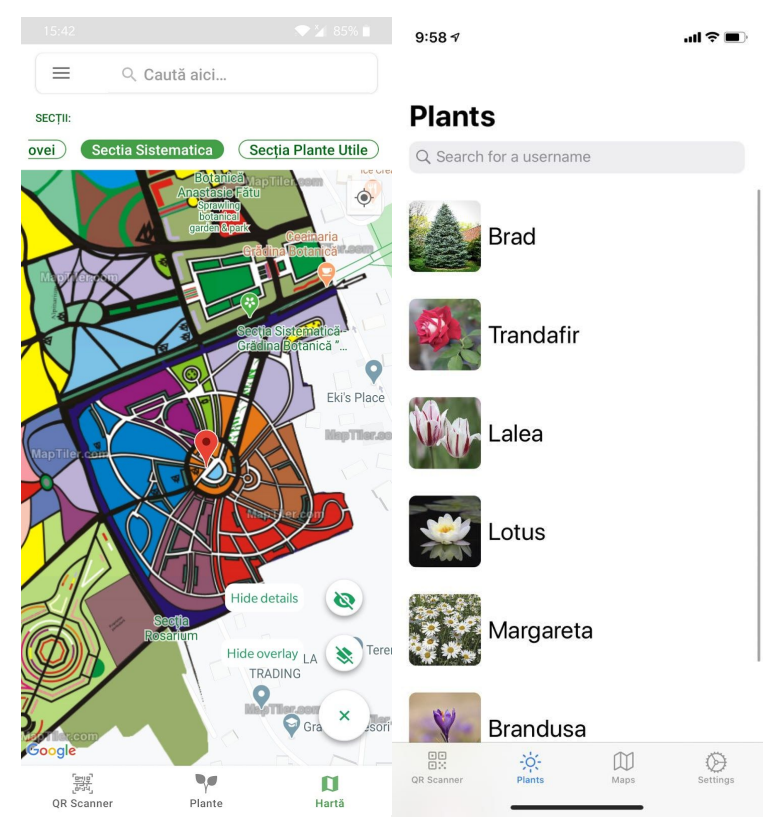

Figure 8. Front page of the "ARGBA" Android App

The user has the option to scan an QR Code to get an AR Model of a Iaşi Botanical Garden supported plant and all the information about it or it can scan any plant to get all the information about it but not the AR Model. Also, the app can give the user directions to the Iaşi Botanical Garden for an interesting virtual tour experience. On the other side, the user can record sounds from surroundings, can trim them and identify any bird supported by the Iaşi Botanical Garden application.

\section{iOS App}

This module came as a necessity because more and more users have a smartphone that runs iOS. This should run as the Android version as much as the platform gives as this capacity. The application will use Apple's ARKit ${ }^{6}$ with the newest SpriteKit ${ }^{7}$ framework and will need user's consent in order to use his Camera or the GPS sensor. Like the Android Application, the iOS one works in a similar way, it has a list of plants, a map with all the sections of the

\footnotetext{
${ }^{6} \mathrm{https}$ ://developer.apple.com/augmented-reality/

${ }^{7}$ https://developer.apple.com/spritekit/
}

Botanical Garden and a QR Scanner that is responsible for recognition of the plates that are all over the garden. The user has also the possibility to change some of the preference settings. Integrated deep into the both applications is the sound recognition module. The user has the possibility to start an audio recording of the surroundings and after he/she has captured the sound of the bird he/she wants to recognize he/she has the ability to crop it and send it to the servers for further processing that will determine which bird he listened to.

\section{Web App}

This module is the Admin part of our application, it is a web app written in Angular ${ }^{8}$ (see Figure 9). This part of the application will be available only for the admin who will create an account based on which they will be authenticated.

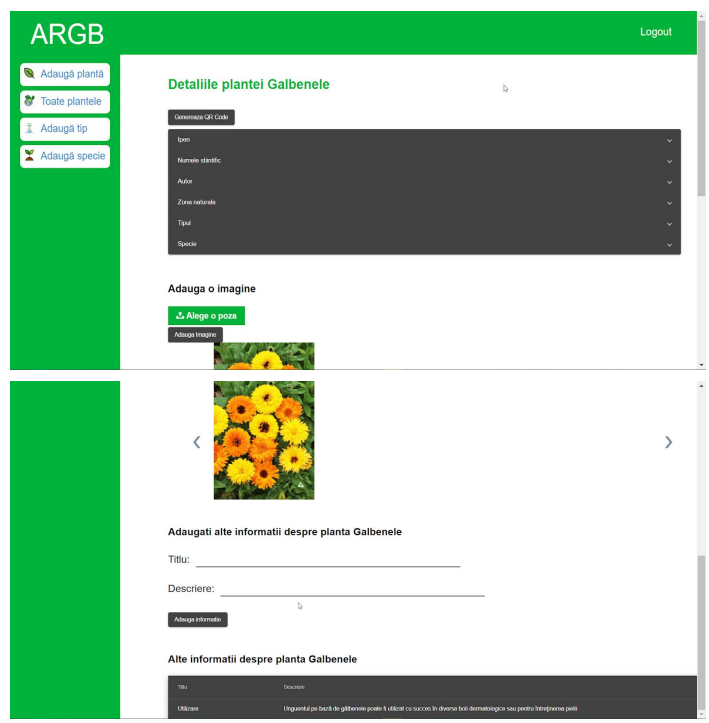

Figure 9. Front page of the Web App "ARGB"

The admin will have the option to add, edit and delete different types of plants and birds that can be found in the Iaşi Botanical Garden. This module has also a submodule to authorize every non-GET call and make the application inaccessible to anyone who is not an admin. The main page of the application has a simple and clean interface that makes interaction as simple and pleasant as possible.

\section{Sound Recognition}

This module is mainly made of an API. The API receives sound recordings and responds with useful information (essentially, the identified species). The app will present the

\footnotetext{
${ }^{8}$ https://angular.io/
} 
received information in an attractive and clear manner. We will consider offering the user the possibility to send feedback to the API. The feedback could be used to improve the trained model or to realize several statistics. Feedback is a nice-to-have feature, as we progress with the app development, we will consider if it is worth introducing it and how it should be used (there are several drawbacks an admin should check the received feedback, it might complicate the interaction between the app and the API, etc.). The module is written in Python and requires several installed modules, such as LibROSA, TensorFlow (with GPU support), Scikit-learn and Flask. It is worth mentioning that the module is developed on Windows and requires a tool, FFmpeg, that enables LibROSA to process more audio formats. There may be similar tools in this context for Windows and other platforms. The mentioned Python modules and their roles in the module are described next.

LibROSA $^{9}$ provides several features in audio analysis. In the context of our module, it extracts features from the audio files and saves them in a format that allows us to do the machine learning work. More exactly, we create mel-spectrograms, with parameter values that are chosen based on the default values in LibROSA or experimentally/empirically. LibROSA allows us to model the spectrograms in great detail. For "reading"/"writing" the mel-spectrograms "from/to" files we used Matplotlib ${ }^{10}$ and NumPy ${ }^{11}$.

TensorFlow ${ }^{12}$ (mostly the included Keras API ${ }^{13}$ ) is used for machine learning tasks. The models are based on neural networks. At installation, we chose an option with support for GPU, in order to get greater speed at various stages in the machine learning processes. The speed is important especially when it comes to the training stage. A trained model is saved and deployed, i.e., it is ready to classify audio recordings received through our API.

Scikit-learn ${ }^{14}$ is used for preprocessing. However, it could

have been used for the actual machine learning processes (but it is more appropriate for other datasets/tasks), as it provides decision trees, support vector machines, etc.

Flask $^{15}$ builds the API itself, as it allows us to specify routes and perform request/response tasks. We were able to

\footnotetext{
${ }^{9}$ https://github.com/librosa/librosa

${ }^{10} \mathrm{https} / / /$ matplotlib.org/

${ }^{11}$ https://numpy.org/

${ }^{12} \mathrm{https}$ ://www.tensorflow.org/

${ }^{13} \mathrm{https}: / /$ keras.io/

${ }^{14} \mathrm{https}: / /$ scikit-learn.org/stable/

${ }^{15} \mathrm{https}$ ://flask.palletsprojects.com/en/1.1.x/
}

decide what the user will send (mainly, sound recordings but additional information may be provided) and what will be the response from the API (the identified species but also relevant information, taken from a taxonomy).

The module is built in such a way that it allows extensions, so in the future it may be used to classify not just birds. However, the extensions require new data, new machine learning processes to be run (essentially, model training) and small configuration changes in the API.

The module is currently "work in progress", as getting a good training dataset is a challenging task. So far, the work (the API and the ML-related processes) has been done on several sample files, provided by the courtesy of Botanical Garden of Iaşi. We intend to get more files, ideally from the Botanical Garden, in order to create a proper dataset and perform the actual training, for a model that can be deployed.

\section{USABILITY TESTING}

In order to be able to analyze whether the objectives listed at the end of the first chapter were achieved, we resorted to performing some usability tests. Usability tests consist of checking a website or a mobile application by potential real users. They analyze elements such as the content, navigation and structure of the application and will note its strengths and weaknesses. In this sense, we have prepared three usage scenarios (described below) that we have offered to several users. After going through these scenarios, we collected their opinions on the application as a whole using a form.

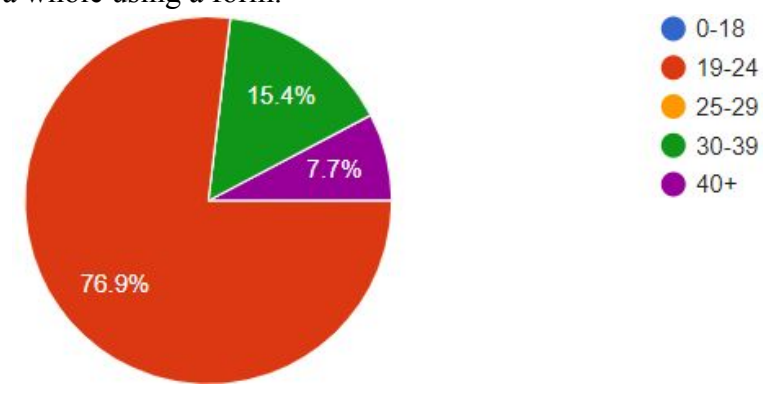

Figure 10. Age distribution of participants in usability tests

In Figure 10 we can see the age distribution of these participants. After completing the three scenarios presented, users were asked to express their opinion on 12 aspects of the application. In the end, we asked them to give an overall rating to the application in its current state, but also to specify how interested they would be in such an application if it had all the functionalities they wanted.

Overall, users were very satisfied with the current way of interacting with the application, the average of the scores 
given by them being over $85 \%$ of the maximum possible for each aspect evaluated separately (see Figure 11). The behavior of the application in each of the scenarios that will be frequently encountered by real users is almost optimal, as can be seen from the first three lines of the other table.

\begin{tabular}{|l|r|r|}
\hline Evaluated aspect & Average & Max \\
\hline Scanning a plant & 5,00 & 5 \\
\hline Searching for information & 4,92 & 5 \\
\hline Using the map & 4,92 & 5 \\
\hline Legibility & 8,08 & 9 \\
\hline Organizing information & 8,46 & 9 \\
\hline Waiting times & 8,92 & 9 \\
\hline Design & 8,08 & 9 \\
\hline Ease of use & 8,38 & 9 \\
\hline Elements on the screen & 8,38 & 9 \\
\hline Navigation in the application & 8,23 & 9 \\
\hline Score in current state & 4,38 & 5 \\
\hline Score if it were further developed & 5,00 & 5 \\
\hline
\end{tabular}

Figure 11. Average scores for evaluated aspects

Data readability, design and navigation within the application are the main elements that should be improved according to the scores obtained. In order to increase readability, we can resort to another type of text alignment (left-right instead of left), highlighting specialized terms by writing them in italics or even providing this information in audio format. The simple and bright design helps a lot to use the application in the outdoor environment, but on devices with small screens, the subtle delimiting elements are no longer easily observable and the elements of the lists appear almost glued. Navigation options are not constant across all application screens. Inserting a "show on map" button in SectionInfoActivity and the menu on the left in all activities and not just in MainActivity would solve this problem.

The most appreciated aspect of the application, according to the scores from Figure 12, was the reduced waiting time. This is due both to the use of paging where possible and to the implementation of the automatic caching system and the encapsulation in Livedata objects of all the data to be used by View so that its drawing is not blocked by their waiting.

Next, we asked participants to specify which are the most useful and least useful features of the application (see Figure 13). The map was considered the most important by almost all participants. This was followed by the QR scanner and the caching system, both of which are elements that help to access information as quickly and easily as possible.

\section{Very useful features}

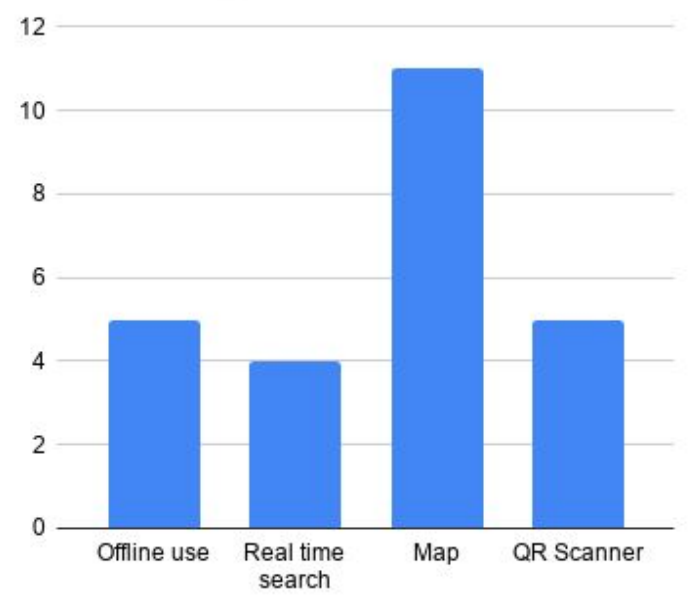

Figure 12. The functionalities considered to be very useful by the participants

\section{Less useful features}

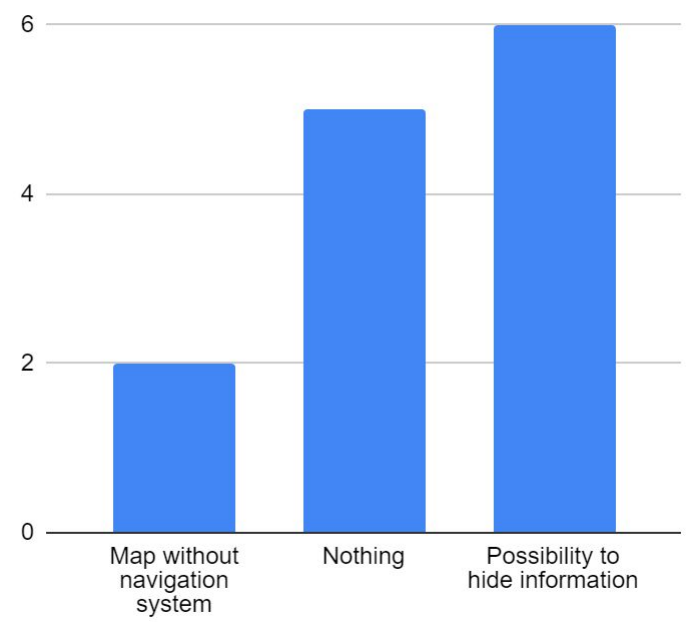

Figure 13. The functionalities considered to be less useful by the participants

In terms of less important functionalities, the possibility to hide information was seen by the participants as being omitted. Their motivation was that, although for a certain plant they consider a type of information to be unimportant and want to hide it, this can lead to the possible loss of interesting data about other plants. At the same time, the map is mentioned here by the participants. This was because these usage tests were done in mid-May, when both the map navigation system using the Google Direction 
API and the AR navigation system were not yet implemented. Thus, at the time of the tests, the functionalities offered by the map were quite limited.

The ability to navigate between points of interest on the map or to traverse routes was specified by almost all participants as a desired element.

\section{CONCLUSION}

In this paper, we presented how augmented reality can be used in the Botanical Garden, and we show details about application developed by us. The application is complex and has multiple modules.

The Web App is the admin part of the application and it is responsible for adding new plants and birds, managing all the existing information and indexing them all in a simple and elegant way. The Android and the iOS apps are responsible for the end-user interaction. Thus, users can scan plants, can search them, find more information and photos about anything in the garden, can scan a mini-table QR Code to receive more information about something, a map of the garden and all its sections and the bird recognition module embedded inside it. The sound recognition module is a machine learning module that can identify over 20 different species of birds and give the user details about it based on a recorded sound. All this considered, we think our application represents a new way to think and to understand better the existing information from the Botanical Garden of Iaşi.

For the future, we intend to improve the existing modules and to increase the resources of the sound recognition module.

\section{REFERENCES}

1. Bederson, B. B. Audio Augmented Reality: A Prototype Automated Tour Guide. CHI'95 Mosaic of Creativity, Short papers (1995), 210-211.

2. Bennett, B. C. Learning in Paradise: The Role of Botanic Gardens in University Education. In Innovative Strategies for Teaching in the Plant Sciences, Springer (2014), 213-229

3. Błaszak, M., Rybska, E., Tsivitanidou, O. and Constantinou, C. P. Botanical Gardens for Productive Interplay between Emotions and Cognition. In MDPI, Concept Paper (2019), 11(24), 7160; https://doi.org/10.3390/su11247160

4. Cherchi, G., Sorrentino, F. and Scaten, R. AR Turn-by-turn navigation in small urban areas and information browsing. STAG: Smart Tools \& Apps for
Graphics. Short papers, Andrea Giachetti (Editor) (2014), 4 pages.

5. Coates, C. How Museums are using Augmented Reality. Museum Next. Digital (2020) https://www.museumnext.com/article/how-museums-a re-using-augmented-reality!

6. Ding, M. Augmented Reality in Museums. Arts Management and Technology Laboratory (2017), 13 pages.

7. Heywood, V. H. Mediterranean botanic gardens and the introduction and conservation of plant diversity. $\mathrm{Fl}$. Medit. 25 (Special Issue) (2015), 103-114, doi: 10.7320/FIMedit25SI.103

8. Karaşah, B. and Var, M. Recreational Functions of Botanical Gardens And Examining Sample of Nezahat Gökyiğit Botanical Garden. In Proceedings of International Caucasian Forestry Symposium (2013), 803-809.

9. Iftene, A. and Trandabăţ, D. Enhancing the Attractiveness of Learning through Augmented Reality. In Proceedings of International Conference on Knowledge Based and Intelligent Information and Engineering Systems, KES2018, 3-5 September 2018, Belgrade, Serbia. Procedia Computer Science 126 (2018), 166-175.

10. Iftene, A., Trandabăţ, D. and Rădulescu, V. Eye and Voice Control for an Augmented Reality Cooking Experience. In 24rd International Conference on Knowledge-Based and Intelligent Information \& Engineering Systems. 16-18 September (2020).

11. Macariu, C., Iftene, A. and Gîfu, D. Learn Chemistry with Augmented Reality. In 24rd International Conference on Knowledge-Based and Intelligent Information \& Engineering Systems. 16-18 September (2020).

12. Păduraru, B. M. and Iftene, A. Tower Defense with Augmented Reality. Proceedings of the 14th Conference on Human Computer Interaction - RoCHI 2017, ISSN 2501-9422, ISSN-L 2501-9422, 11-12 September 2017, Craiova, Romania (2017), 113-118.

13. Pinzariu, M. N. and Iftene, A. Sphero - Multiplayer Augmented Game (SMAUG). In International Conference on Human-Computer Interaction, 8-9 September 2016, Iasi, Romania (2016), 46-49.

14. Porfireanu, A., Ungurean, A., Dascălu, A., Iftene, A. and Gîfu, D. Smart Museums with Augmented Reality. In 5th Proceedings of the Conference on Mathematical Foundations of Informatics. 3-6 July 2019, Iasi, Romania (2019), 285-294. 